\title{
A SPARSITY RESULT FOR THE DYNAMICAL MORDELL-LANG CONJECTURE IN POSITIVE CHARACTERISTIC
}

\author{
DRAGOS GHIOCA, ALINA OSTAFE, SINA SALEH, \\ AND IGOR E. SHPARLINSKI
}

\begin{abstract}
We prove a quantitative partial result in support of the Dynamical Mordell-Lang Conjecture (also known as the $D M L$ conjecture) in positive characteristic. More precisely, we show the following: given a field $K$ of characteristic $p$, given a semiabelian variety $X$ defined over a finite subfield of $K$ and endowed with a regular self-map $\Phi: X \longrightarrow X$ defined over $K$, given a point $\alpha \in X(K)$ and a subvariety $V \subseteq X$, then the set of all non-negative integers $n$ such that $\Phi^{n}(\alpha) \in V(K)$ is a union of finitely many arithmetic progressions along with a subset $S$ with the property that there exists a positive real number $A$ (depending only on $N$, $\Phi, \alpha, V)$ such that for each positive integer $M$, we have
\end{abstract}

$$
\#\{n \in S: n \leq M\} \leq A \cdot(1+\log M)^{\operatorname{dim} V} .
$$

\section{IntroduCtion}

1.1. Notation. Throughout this paper, we let $\mathbb{N}_{0}:=\mathbb{N} \cup\{0\}$ denote the set of nonnegative integers. As always in arithmetic dynamics, we denote by $\Phi^{n}$ the $n$-th iterate of the self-map $\Phi$ acting on some ambient variety $X$. For each point $x$ of $X$, we denote its orbit under $\Phi$ by

$$
\mathcal{O}_{\Phi}(x):=\left\{\Phi^{n}(x): n \in \mathbb{N}_{0}\right\} .
$$

Also, for us, an arithmetic progression is a set $\{a n+b\}_{n \in \mathbb{N}_{0}}$ for some $a, b \in \mathbb{N}_{0}$; in particular, we allow the possibility that $a=0$, in which case, the above set is a singleton.

1.2. The Dynamical Mordell-Lang Conjecture. The Dynamical Mordell-Lang Conjecture (see [GT09]) predicts that for an endomorphism $\Phi$ of a quasiprojective variety $X$ defined over a field $K$ of characteristic 0 , given a point $\alpha \in X(K)$ and a subvariety $V \subseteq X$, the

2010 Mathematics Subject Classification. Primary 11B37, Secondary 11G25, $37 \mathrm{P} 55$.

Key words and phrases. linear recurrence sequences, Dynamical Mordell-Lang Conjecture. 
set

$$
\mathcal{S}(\Phi, \alpha ; V):=\left\{n \in \mathbb{N}_{0}: \Phi^{n}(\alpha) \in V(K)\right\}
$$

is a finite union of arithmetic progressions; for a comprehensive discussion of the Dynamical Mordell-Lang Conjecture, we refer the reader to the book [BGT16].

When the field $K$ has positive characteristic, then under the same setting as above, the return set $\mathcal{S}$ from (1.1) is no longer a finite union of arithmetic progressions, as shown in [Ghi19, Examples 1.2 and 1.4]; instead, the following conjecture is expected to hold.

Conjecture 1.1 (Dynamical Mordell-Lang Conjecture in positive characteristic). Let $X$ be a quasiprojective variety defined over a field $K$ of characteristic $p$. Let $\alpha \in X(K)$, let $V \subseteq X$ be a subvariety defined over $K$, and let $\Phi: X \longrightarrow X$ be an endomorphism defined over $K$. Then the set $\mathcal{S}(\Phi, \alpha ; V)$ given by (1.1) is a union of finitely many arithmetic progressions along with finitely many sets of the form

$$
\left\{\sum_{j=1}^{m} c_{j} p^{a_{j} k_{j}}: k_{j} \in \mathbb{N}_{0} \text { for each } j=1, \ldots m\right\},
$$

for some given $m \in \mathbb{N}$, some given $c_{j} \in \mathbb{Q}$, and some given $a_{j} \in$ $\mathbb{N}_{0}$ (note that in (1.2), the parameters $c_{j}$ and $a_{j}$ are fixed, while the unknowns $k_{j}$ vary over all non-negative integers, $\left.j=1, \ldots, m\right)$.

In [CGSZ20], Conjecture 1.1 is proven for regular self-maps $\Phi$ of tori assuming one of the following two hypotheses are met:

(A) $\operatorname{dim} V \leq 2$;

or

(B) $\Phi: \mathbb{G}_{m}^{N} \longrightarrow \mathbb{G}_{m}^{N}$ is a group endomorphism and there exists no nontrivial connected algebraic subgroup $G$ of $\mathbb{G}_{m}^{N}$ such that an iterate of $\Phi$ induces an endomorphism of $G$ that equals a power of the usual Frobenius.

The proof from [CGSZ20] employs various techniques from Diophantine approximation (in characteristic 0 ), to combinatorics over finite fields, to specific tools akin to semiabelian varieties defined over finite fields; in particular, the deep results of Moosa \& Scanlon [MS04] are essential in the proof. Actually, the Dynamical Mordell-Lang Conjecture in positive characteristic turns out to be even more difficult than the classical Dynamical Mordell-Lang Conjecture since even the case of group endomorphisms of $\mathbb{G}_{m}^{N}$ leads to deep Diophantine questions in characteristic 0, as shown in [CGSZ20, Theorem 1.4]. More precisely, [CGSZ20, Theorem 1.4] shows that solving Conjecture 1.1 just 
in the case of group endomorphisms of tori is equivalent with solving the following polynomial-exponential equation: given any linear recurrence sequence $\left\{u_{n}\right\}$, given a power $q$ of the prime number $p$, and given positive integers $c_{1}, \ldots, c_{m}$ such that

$$
\sum_{i=1}^{m} c_{i}<\frac{q}{2},
$$

then one needs to determine the set of all $n \in \mathbb{N}_{0}$ for which we can find $k_{1}, \ldots, k_{m} \in \mathbb{N}_{0}$ such that

$$
u_{n}=\sum_{i=1}^{m} c_{i} q^{k_{i}} .
$$

The equation (1.3) remains unsolved for general sequences $\left\{u_{n}\right\}$ when $m>2$; for more details about these Diophantine problems, see [CZ13] and the references therein.

1.3. Statement of our results. Before stating our main result, we recall that a semiabelian variety is an extension of an abelian variety by an algebraic torus; for more details on semiabelian varieties, we refer the reader to [CGSZ20, Section 2.1] and the references therein.

We prove the following result towards Conjecture 1.1.

Theorem 1.2. Let $K$ be a field of characteristic $p$, let $X$ be a semiabelian variety defined over a finite subfield of $K$, let $\Phi$ be a regular self-map of $X$ defined over $K$. Let $V \subseteq X$ be a subvariety defined over $K$ and let $\alpha \in X(K)$. Then the set $\mathcal{S}(\Phi, \alpha ; V)$ defined by (1.1) is a union of finitely many arithmetic progressions along with a set $S \subseteq \mathbb{N}_{0}$ for which there exists a constant $A$ depending only on $X, \Phi, \alpha$ and $V$ such that for all $M \in \mathbb{N}$, we have

$$
\#\{n \in S: n \leq M\} \leq A \cdot(1+\log M)^{\operatorname{dim} V} .
$$

Our result strengthens [BGT15, Corollary 1.5] for the case of regular self-maps of semiabelian varieties defined over finite fields since in [BGT15] it is shown that the set $S$ (as in the conclusion of Theorem 1.2) is of Banach density zero; however, the methods from [BGT15] cannot be used to obtain a sparseness result as the one from (1.4).

We establish Theorem 1.2 by combining [CGSZ20, Theorem 3.2] with [Lau84, Théorème 6].

\section{Proof of Theorem 1.2}

\subsection{Dynamical Mordell-Lang conjecture and linear recurrence}

sequences. First, since $X$ is defined over a finite field $\mathbb{F}_{q}$ of $q$ elements 
of characteristic $p$, we let $F: X \longrightarrow X$ be the Frobenius endomorphism corresponding to $\mathbb{F}_{q}$. We let $P \in \mathbb{Z}[x]$ be the minimal polynomial with integer coefficients such that $P(F)=0$ in $\operatorname{End}(X)$; according to [CGSZ20, Section 2.1], $P$ is a monic polynomial and it has simple roots $\lambda_{1}, \ldots, \lambda_{\ell}$, each one of them of absolute value equal to $q$ or $\sqrt{q}$.

Using [CGSZ20, Theorem 3.2], we obtain that the set $\mathcal{S}(\Phi, \alpha ; V)$ defined by (1.1) is a finite union of generalized $F$-arithmetic sequences, and furthermore, each such generalized $F$-arithmetic sequence is an intersection of finitely many $F$-arithmetic sequences; see [CGSZ20, Section 3] for exact definitions. Each one of these $F$-arithmetic sequences consists of all non-negative integers $n$ belonging to a suitable arithmetic progression, for which there exist $k_{1}, \ldots, k_{m} \in \mathbb{N}_{0}$ such that

$$
u_{n}=\sum_{i=1}^{m} \sum_{j=1}^{\ell} c_{i, j} \lambda_{j}^{a_{i} k_{i}}
$$

for some given linear recurrence sequence $\left\{u_{n}\right\} \subset \overline{\mathbb{Q}}$, some given $m, \ell \in$ $\mathbb{N}$, some given constants $c_{i, j} \in \overline{\mathbb{Q}}$ and some given $a_{1}, \ldots, a_{m} \in \mathbb{N}$. Applying Part (1) of [CGSZ20, Theorem 3.2], we also see that $m \leq$ $\operatorname{dim} V$. Furthermore, the linear recurrence sequence $\left\{u_{n}\right\}$ along with the constants $c_{i, j}$ and $a_{j}$ depend solely on $X, \Phi, \alpha$ and $V$.

Moreover, at the expense of further refining to another arithmetic progression, we may assume from now on, that the linear recurrence sequence $\left\{u_{n}\right\}$ is non-degenerate, i.e the quotient of any two characteristic roots of this linear recurrence sequence is not a root of unity; furthermore, we may also assume that if one of the characteristic roots is a root of unity, then it actually equals 1 . For more details regarding linear recurrence sequences, we refer the reader to [Sch03]. In addition, we know that the characteristic roots of $\left\{u_{n}\right\}$ are all algebraic integers (see part (2) of [CGSZ20, Theorem 3.2]); the characteristic roots of $\left\{u_{n}\right\}$ are either equal to 1 (when $\Phi$ contains also a translation besides a group endomorphism) or equal to positive integer powers of the roots of the minimal polynomial of $\Phi$ inside $\operatorname{End}(X)$; for more details, see [CGSZ20, Section 3]. So, the equation (2.1) becomes

$$
\sum_{r=1}^{s} Q_{r}(n) \mu_{r}^{n}=\sum_{i=1}^{m} \sum_{j=1}^{\ell} c_{i, j} \lambda_{j}^{a_{i} k_{i}}
$$

where $\mu_{1}, \ldots, \mu_{s}$ are the characteristic roots of the sequence $\left\{u_{n}\right\}$ and $Q_{1}, \ldots, Q_{s} \in \overline{\mathbb{Q}}[x]$. 
2.2. Reduction to the case $s=1$. Now, if each polynomial $Q_{r}$ from the equation (2.2) is constant, then the famous result of Laurent [Lau84] solving the classical Mordell-Lang conjecture (inside an algebraic torus) provides the desired conclusion that the set of all $n \in \mathbb{N}_{0}$ satisfying an equation of the form (2.2) must be a finite union of arithmetic progressions. So, from now on, we assume that not all of the polynomials $Q_{r}$ are constant.

Without loss of generality, we assume $Q_{1}$ is a non-constant polynomial. According to [Lau84, Section 8, p. 319] (see also [Sch03, Theorem 7.1]) all but finitely many solutions to the equation (2.2) are also solutions to a subsum corresponding to the equation (2.2) which contains the term $Q_{1}(n) \mu_{1}^{n}$. More precisely, there exists a subset $1 \in \Sigma_{1} \subseteq$ $\{1, \ldots, s\}$ and also, there exists a subset $\Sigma_{2} \subseteq\{1, \ldots, m\} \times\{1, \ldots, \ell\}$ such that

$$
\sum_{r \in \Sigma_{1}} Q_{r}(n) \mu_{r}^{n}=\sum_{(i, j) \in \Sigma_{2}} c_{i, j} \lambda_{j}^{a_{i} k_{i}} .
$$

Moreover, letting $\pi_{1}:\{1, \ldots, m\} \times\{1, \ldots, \ell\} \longrightarrow\{1, \ldots, m\}$ be the projection on the first coordinate, we have $m_{1}:=\#\left(\pi_{1}\left(\Sigma_{2}\right)\right)$; in particular, $m_{1} \leq m$. Without loss of generality, we assume $\pi_{1}\left(\Sigma_{2}\right)=$ $\left\{1, \ldots, m_{1}\right\}$ (with the understanding that, a priori, $m_{1}$ could be equal to 0 , even though we show next that this is not the case).

Using [Lau84, Théorème 6], the equation (2.3) has finitely many solutions, unless the following subgroup $G_{\Sigma} \subseteq \mathbb{Z}^{1+m_{1}}$ is nontrivial. As described in [Lau84, Section 8, p. 320], the subgroup $G_{\Sigma}$ consists of all tuples $\left(f_{0}, f_{1}, \ldots, f_{m_{1}}\right)$ of integers with the property that

$$
\mu_{r}^{f_{0}}=\lambda_{j}^{a_{i} f_{i}} \text { for each } r \in \Sigma_{1} \text { and each }(i, j) \in \Sigma_{2} .
$$

Since $\mu_{r_{2}} / \mu_{r_{1}}$ is not a root of unity if $r_{1} \neq r_{2}$, we conclude that if $\Sigma_{1}$ contains at least two elements (we already have by our assumption that $1 \in \Sigma_{1}$ ), then $f_{0}=0$ in (2.4); furthermore, if $f_{0}=0$, then the equation (2.4) yields that each $f_{i}=0$ (since each $\lambda_{j}$ has an absolute value greater than 1 and $a_{i} \in \mathbb{N}$ ). So, if $\Sigma_{1}$ has more than one element, then the subgroup $G_{\Sigma}$ is trivial and thus, [Lau84, Théorème 6] yields that the equation (2.3) (and therefore, also the equation (2.2)) has finitely many solutions, as desired.

2.3. Concluding the argument. Therefore, from now on, we may assume that $\Sigma_{1}$ has a single element, i.e., $\Sigma_{1}=\{1\}$. In particular, this also means that $\Sigma_{2}$ cannot be the empty set since otherwise the equation (2.3) would simply read

$$
Q_{1}(n) \mu_{1}^{n}=0
$$


which would only have finitely many solutions $n$ (since $\mu_{1} \neq 0$ and $Q_{1}$ is non-constant). So, we see that indeed $\Sigma_{2}$ is nonempty, which also means that $1 \leq m_{1} \leq m$.

We have two cases: either $\mu_{1}$ equals 1 , or not.

Case 1. $\mu_{1}=1$.

Then the equation (2.3) reads:

$$
Q_{1}(n)=\sum_{(i, j) \in \Sigma_{2}} c_{i, j} \lambda_{j}^{a_{i} k_{i}}
$$

Now, for the equation (2.5), the subgroup $G_{\Sigma}$ defined above as in [Lau84, Section 8, p. 320] is the subgroup $\mathbb{Z} \times\{(0, \ldots, 0)\} \subset \mathbb{Z}^{1+m_{1}}$ since each integer $f_{i}$ from the equation (2.4) must equal 0 for $i=1, \ldots, m_{1}$ (note that $\mu_{1}=1$, while each $\lambda_{j}$ is not a root of unity). According to [Lau84, Théorème 6 , part (b)], there exist positive constants $A_{1}$ and $A_{2}$ depending only on $Q_{1}$, the $c_{i, j}$ and the $a_{i}$ such that for any solution $\left(n, k_{1}, \ldots, k_{m_{1}}\right)$ of the equation $(2.5)$, we have

$$
\max \left\{\left|k_{1}\right|, \ldots,\left|k_{m_{1}}\right|\right\} \leq A_{1} \log |n|+A_{2} .
$$

So, for each non-negative integer $n \leq M$ (for some given upper bound M) for which there exist integers $k_{i}$ satisfying the equation (2.5), we have that $\left|k_{i}\right| \leq A_{2}+A_{1} \log M$, which means that we have at most $A_{3}(1+\log M)^{m_{1}}$ possible tuples $\left(k_{1}, \ldots, k_{m_{1}}\right) \in \mathbb{Z}^{m_{1}}$, which may correspond to some $n \in\{0, \ldots, M\}$ solving the equation (2.5) (where, once again, $A_{3}$ is a constant depending only on the initial data in our problem). Since $Q$ is a polynomial of degree $D \geq 1$, we conclude that the number of solutions $0 \leq n \leq M$ to the equation (2.5) is bounded above by $D \cdot A_{3}(1+\log M)^{m_{1}}$. Finally, recalling that $m_{1} \leq m \leq \operatorname{dim} V$, we obtain the desired conclusion from inequality (1.4).

Case 2. $\mu_{1} \neq 1$.

In this case, since we also know that any characteristic root $\mu_{r}$ of the linear reccurence sequence $\left\{u_{n}\right\}_{n \in \mathbb{N}_{0}}$ is either equal to 1 , or not a root of unity, we conclude that $\mu_{1}$ is not a root of unity.

The equation (2.3) reads now:

$$
Q_{1}(n) \mu_{1}^{n}=\sum_{(i, j) \in \Sigma_{2}} c_{i, j} \lambda_{j}^{a_{i} k_{i}}
$$

We analyze again the subgroup $G_{\Sigma} \subseteq \mathbb{Z}^{1+m_{1}}$ containing the tuples $\left(f_{0}, f_{1}, \ldots, f_{m_{1}}\right)$ of integers satisfying the equations $(2.4)$, i.e.,

$$
\mu_{1}^{f_{0}}=\lambda_{j}^{a_{i} f_{i}} \text { for each }(i, j) \in \Sigma_{2} .
$$

Because $\mu_{1}$ is not a root of unity and also each $\lambda_{j}$ is not a root of unity, while the $a_{i}$ are positive integers, we conclude that a nontrivial tuple 
$\left(f_{0}, f_{1}, \ldots, f_{m_{1}}\right)$ satisfying the equations (2.8) must actually have each entry nonzero (i.e., $f_{i} \neq 0$ for each $i=0, \ldots, m_{1}$ ). Therefore, each $\lambda_{j}^{a_{i}}$ is multiplicatively dependent with respect to $\mu_{1}$ and so, there exists an algebraic number $\lambda$ (which is not a root of unity), there exists a nonzero integer $b$ such that $\mu_{1}=\lambda^{b}$, and whenever there is a pair $(i, j) \in \Sigma_{2}$, there exist roots of unity $\zeta_{j, i}$ along with nonzero integers $b_{i}$ such that

$$
\lambda_{j}^{a_{i}}=\zeta_{j, i} \cdot \lambda^{b_{i}} .
$$

We let $E$ be a positive integer such that $\zeta_{j, i}^{E}=1$ for each $(j, i) \in \Sigma_{2}$; then we let $B_{i}:=E \cdot b_{i}$ for each $i=1, \ldots, m_{1}$. We now put each exponent $k_{i}$ appearing in (2.7) in a prescribed residue class modulo $E$ (just getting $E^{m}$ possible choices) and use (2.9) along with the fact that $\mu_{1}=\lambda^{b}$. Writing $K_{i}:=\left\lfloor k_{i} / E\right\rfloor, i=1, \ldots, m_{1}$, we obtain that finding $n \in \mathbb{N}_{0}$ which solves the equation (2.7) (and then, in turn, also (2.3) and (2.2)) reduces to finding $n \in \mathbb{N}_{0}$ which solves at least one of the at most $E^{m}$ distinct equations of the form:

$$
Q_{1}(n) \lambda^{b n}=\sum_{i=1}^{m_{1}} d_{i} \lambda^{B_{i} K_{i}},
$$

for some algebraic numbers $d_{1}, \ldots, d_{m_{1}}$, depending only on $E$, the $c_{i}$, and the $\zeta_{j, i},(i, j) \in \Sigma_{2}$. So, dividing the equation (2.10) by $\lambda^{b n}$ yields that

$$
Q_{1}(n)=\sum_{i=1}^{m_{1}} d_{i} \lambda^{g_{i}},
$$

for some integers $g_{i}$. Then once again applying [Lau84, Théorème 6, part (b)] (see also our inequality (2.6)) yields immediately that any solution $\left(n, g_{1}, \ldots, g_{m_{1}}\right)$ to the equation (2.11) must satisfy the inequality:

$$
\max \left\{\left|g_{1}\right|, \ldots,\left|g_{m_{1}}\right|\right\} \leq A_{4} \log |n|+A_{5},
$$

for some constants $A_{4}$ and $A_{5}$ depending only on the initial data in our problem $(X, \Phi, \alpha, V)$. Then once again (exactly as in Case $\mathbf{1}$ ), we conclude that there exists a constant $A_{6}$ such that for any given upper bound $M \in \mathbb{N}$, we have at most $A_{6}(1+\log M)^{m_{1}}$ possible tuples $\left(g_{1}, \ldots, g_{m_{1}}\right) \in \mathbb{Z}^{m_{1}}$, which may correspond to some $n \in\{0, \ldots, M\}$ solving the equation (2.11). Since $Q$ is a polynomial of degree $D \geq 1$, we conclude that the number of solutions $0 \leq n \leq M$ to the equation (2.11) is bounded above by $D \cdot A_{6}(1+\log M)^{m_{1}}$. Finally, recalling that $m_{1} \leq m \leq \operatorname{dim} V$, we obtain the desired conclusion from inequality (1.4).

This concludes our proof of Theorem 1.2. 


\section{Comments}

Remark 3.1. If in the equation (2.2) there exists at least one characteristic root $\mu_{r}$ of $\left\{u_{n}\right\}$ which is multiplicatively independent with respect to each one of the $\lambda_{j}$, then there is never a subsum (2.3) containing $\mu_{r}$ on its left-hand side for which the corresponding group $G_{\Sigma}$ would be nontrivial. So, in this case, the equation (2.2) would have only finitely many solutions. Therefore, with the notation as in Theorem 1.2, arguing as in the proof of [CGSZ20, Theorem 1.3], one concludes that if $\Phi$ is a group endomorphism of the semiabelian variety $X$ with the property that each characteristic root of its minimal polynomial (in $\operatorname{End}(X)$ ) is multiplicatively independent with respect to each eigenvalue $\lambda_{j}$ of the Frobenius endomorphism of $X$, then for each $\alpha \in X(K)$, the set $\mathcal{S}(\Phi, \alpha ; V)$ defined by $(1.1)$ is a finite union of arithmetic progressions.

Remark 3.2. We notice that in (2.11), if we deal with a polynomial $Q$ of degree 1, then the conclusion from inequality (1.4) is sharp. More precisely, as a specific example, the number of positive integers $n \leq M$ which have precisely $m$ nonzero digits (all equal to 1 ) in base- $p$ is of the order of $(\log M)^{m}$, which shows that Theorem 1.2 is tight if the Dynamical Mordell-Lang Conjecture reduces to solving the equation (2.11) when $Q(n)=n, c_{1}=\ldots=c_{m}=1$ and $\lambda=p$. As proven in [CGSZ20, Theorem 1.4], there are instances when the Dynamical Mordell-Lang Conjecture reduces precisely to such equation.

Now, for higher degree polynomials $Q \in \mathbb{Z}[x]$ appearing in the equation (2.11), one expects a lower exponent than $m$ appearing in the upper bounds from (1.4). One also notices that for any polynomial $Q$, arguments $n$ with $k$ nonzero digits in base- $p$ lead to sparse outputs. Hence, simple combinatorics allows us to obtain a lower bound on the best possible exponent in (1.4). However, finding a more precise exponent replacing $m$ in (1.4) when $\operatorname{deg} Q>1$ seems very difficult beyond some special cases; the authors hope to return to this problem in a sequel paper.

\section{ACKNOWLEDGEMENT}

D. G. and S S. were partially supported by a Discovery Grant from NSERC, A. O. by ARC Grants DP180100201 and DP200100355, and I. S. by an ARC Grant DP200100355.

\section{REFERENCES}

[BGT15] J. P. Bell, D. Ghioca, and T. J. Tucker, The Dynamical Mordell-Lang problem for Noetherian spaces, Funct. Approx. Comment. Math. 53 (2015), 313-328. 3 
[BGT16] J. P. Bell, D. Ghioca, and T. J. Tucker, The dynamical Mordell-Lang conjecture, Mathematical Surveys and Monographs, 210, American Mathematical Society, Providence, RI, 2016, xiii+280 pp. 2

[CGSZ20] P. Corvaja, D. Ghioca, T. Scanlon, and U. Zannier, The Dynamical Mordell-Lang Conjecture for endomorphisms of semiabelian varieties defined over fields of positive characteristic, J. Inst. Math. Jussieu, to appear. $2,3,4,8$

[CZ13] P. Corvaja and U. Zannier, Finiteness of odd perfect powers with four nonzero binary digits, Ann. Inst. Fourier (Grenoble) 63 (2013), 715-731. 3

[Ghi19] D. Ghioca, The dynamical Mordell-Lang conjecture in positive characteristic, Trans. Amer. Math. Soc. 371 (2019), 1151-1167. 2

[GT09] D. Ghioca and T. J. Tucker, Periodic points, linearizing maps, and the dynamical Mordell-Lang problem, J. Number Theory 129 (2009), 13921403. 1

[Lau84] M. Laurent, Équations diophantiennes exponentielles, Invent. Math. 78 (1984), 299-327. 3, 5, 6, 7

[MS04] R. Moosa and T. Scanlon, F-structures and integral points on semiabelian varieties over finite fields, Amer. J. Math. 126 (2004), 473-522. 2

[Sch03] W. Schmidt, Linear recurrence sequences, Diophantine Approximation (Cetraro, Italy, 2000), Lecture Notes in Math. 1819, Springer-Verlag Berlin Heidelberg, 2003, pp. 171-247. 4, 5

Dragos Ghioca, Department of Mathematics, University of British Columbia, Vancouver, BC V6T 1Z2, Canada

Email address: dghioca@math.ubc.ca

Alina Ostafe, School of Mathematics and Statistics, University of New South Wales, Sydney NSW 2052, Australia

Email address: alina.ostafe@unsw.edu.au

Sina Saleh, Department of Mathematics, University of British CoLumbia, Vancouver, BC V6T 1Z2, Canada

Email address: sinas@math.ubc.ca

Igor E. Shparlinski, School of Mathematics and Statistics, UniverSity of New South Wales, Sydney NSW 2052, Australia

Email address: igor.shparlinski@unsw.edu.au 